\title{
Electron beam controlled ice nucleation behavior at low temperature
}

Yulin Lin ${ }^{1}$, Xiao-min Lin $^{2}$, Aiwen Lei ${ }^{3}$ and Jianguo Wen ${ }^{2}$

${ }^{1}$ Institute of Advanced Studies, Wuhan University, Wuhan, Hubei, China (People's Republic), ${ }^{2}$ Argonne National Laboratory, Lemont, Illinois, United States, ${ }^{3}$ Wuhan University, Lemont, Illinois, United States

Vapor-deposited water ice plays a critical role in various natural phenomena. The crystalline phase of ice grown from the water vapor primarily depends on the growth temperature and pressures [1]. The water partial pressure in a high vacuum TEM chamber is extremely low, so the ice condensation occurs under a low temperature such as $100 \mathrm{~K}$, which leads to the formation of cubic ice. With further decreasing the deposition temperatures to $\sim 40 \mathrm{~K}$, amorphous ice can be observed [2]. On the other hand, the surface property also affects the nucleation behavior of ice dramatically. By treating the surface coated by ice with electron beam irradiation, we obtain amorphous ice under the condition that originally prefers to form cubic ice. This method is mainly applied to the carbon materials such as amorphous carbon, diamond, carbon nanotubes (CNTs).

This surface treatment requires a combination of ice and a strong electron beam. At $100 \mathrm{~K}$, the residual water vapor in the TEM chamber forms cubic nanoparticles fully covered the surface of CNTs (Fig. 1(a)). Under an intense $200 \mathrm{keV}$ electron beam irradiation ( 104 e-/Å2s), the cubic ice sublimates in 30 seconds, exposing the clean surface of CNTs (Fig. 1(b)). About 30 minutes later without electron beam irradiation, a new ice layer with a totally different morphology is formed at the irradiated area (Fig. 1(c)). The newly formed ice uniformly covered the surface of the CNTs without an obvious particle shape. Fig. 1(d) shows a low-dose HRTEM image ( 103 e-/Å2s) and corresponding FFT pattern of a cubic nanoparticle taking with a Gatan direct detection camera. In contrast, there is no lattice observed from the newly formed ice. SEAD pattern of originally formed ice at $100 \mathrm{~K}$ is indexed as a typical cubic structure with bright diffraction spots (Fig. 1(e)). The SEAD in Fig. 1(f) of the newly formed ice with illegible fuzzy diffraction rings indicates the formation of amorphous ice. The fine structure of the O-K edge is also used to tell the structure difference between crystalline and amorphous ice. As shown in Fig. 2(a), the intensity ratio between peak III and II of cubic ice is higher than the amorphous ice in the O-K edge EELS spectra, further confirm a non-crystalline ice structure was formed after the irradiation. This method is successfully applied to several other carbon materials such as amorphous carbon Fig. 2(b-c), diamond nanoparticles Fig. 2(d-e). The amorphous ice can form along the surface and fits with various shape, indicating the contact angle of the amorphous ice nucleus between the substrate is near to zero. The surface of carbon materials becomes more hydrophilic after the interaction with ice and the electron beam irradiation. Ice is a weak oxidant at low temperatures. But the oxygen or oxygen radicals formed during the ionization of ice under the electron beam irradiation may introduce hydrophilic groups on the carbon surface [3], which reduces the surface free energy and lead to the formation of amorphous ice. [4] 

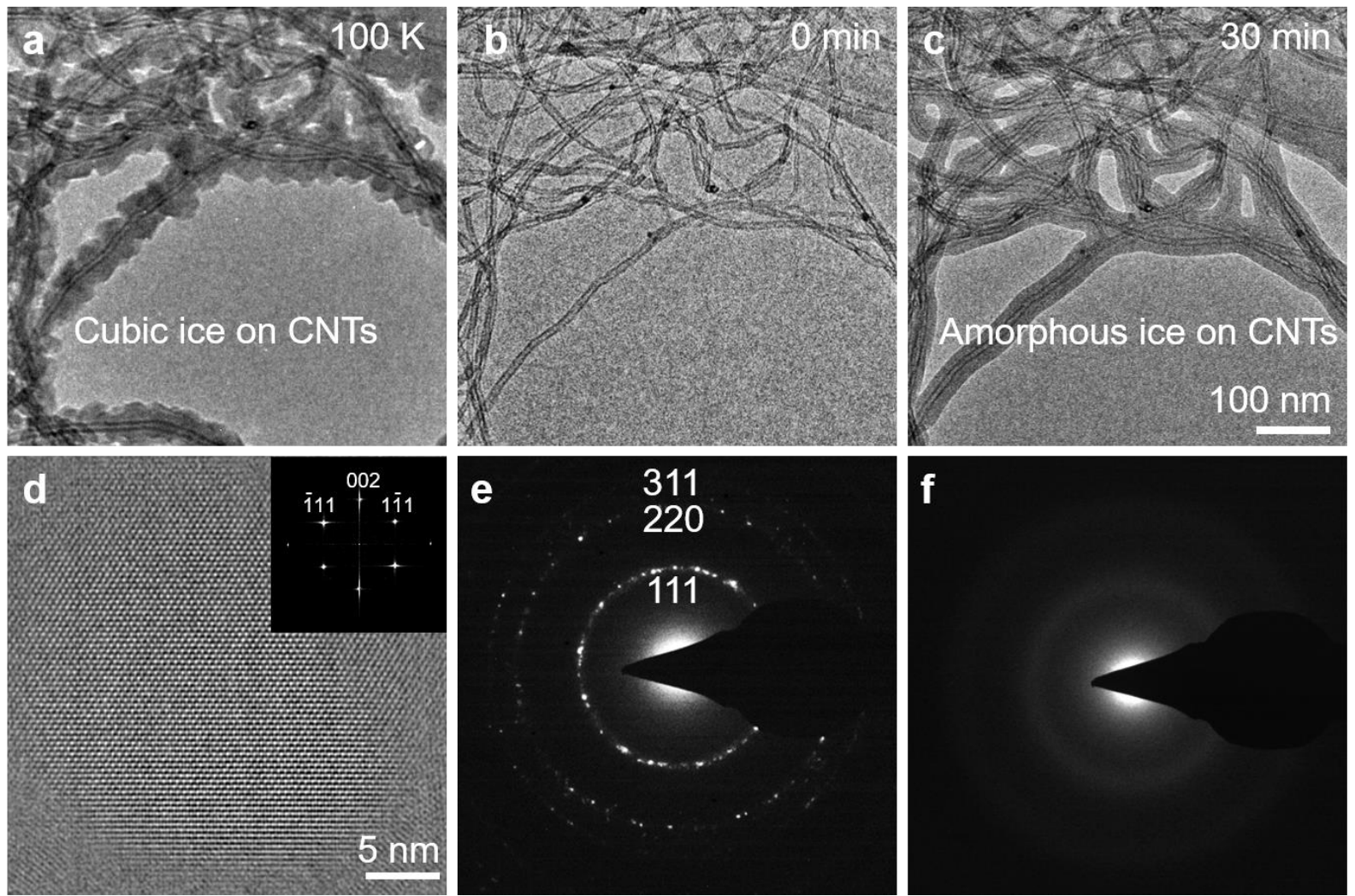

Figure 1. Fig 1. (a) CNTs covered by the cubic ice (b) clean CNTs deicing by electron beam irradiation (c) CNTs covered by the amorphous ice (d) HRTEM and corresponding FFT pattern of a pure cubic ice nanoparticle. (e) Typical SAED pattern of the cubic ice on the amorphous carbon film (e) Typical SAED pattern of the amorphous ice on the amorphous carbon film.
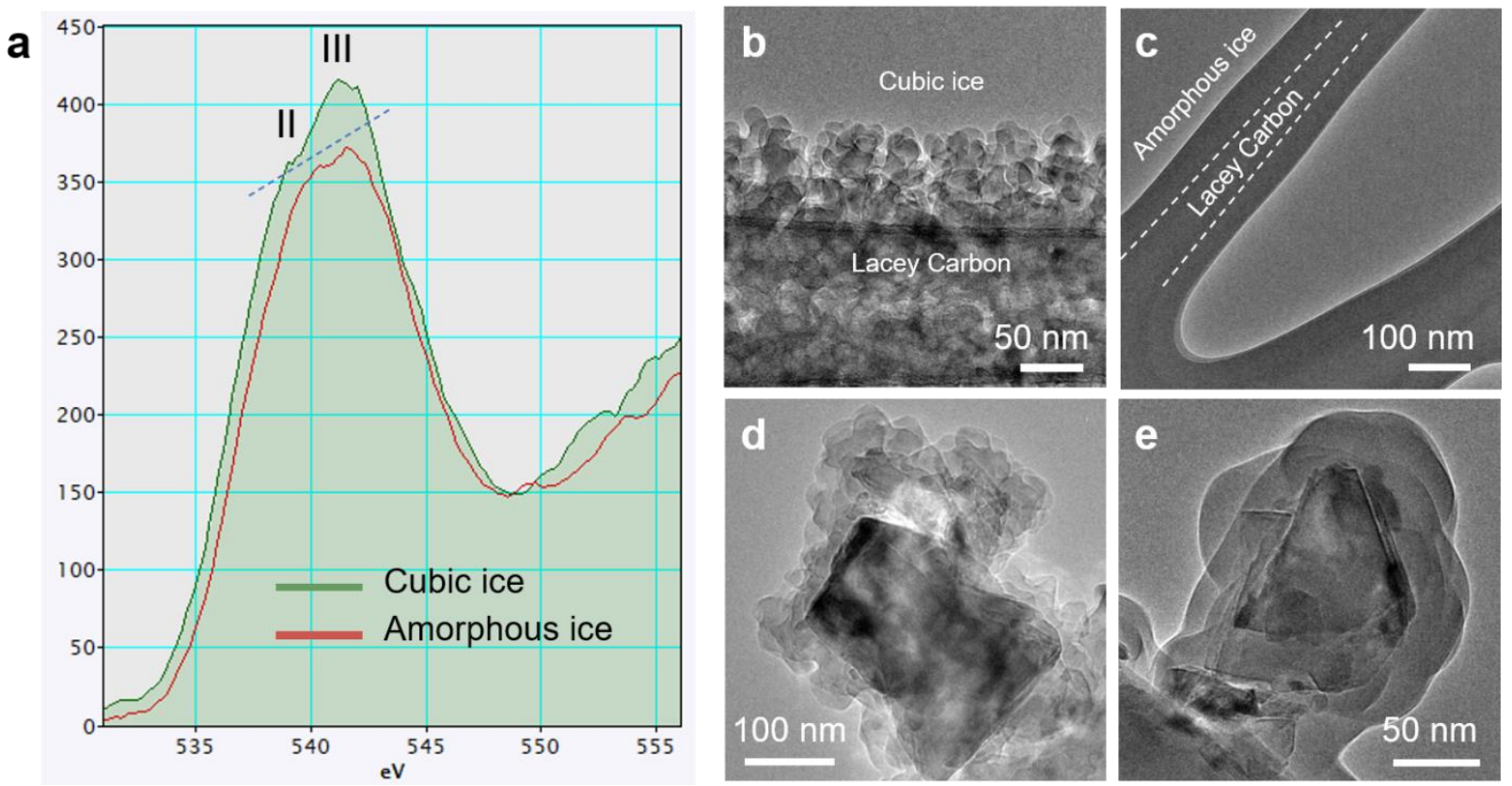

Figure 2. Fig 2. (a) Oxygen core-loss spectra of cubic ice and amorphous ice (b) Lacey carbon frame covered by cubic ice (c) Lacey carbon frame covered by amorphous ice (d) diamond nanoparticles covered by cubic ice (e) diamond nanoparticles covered by amorphous ice. 


\section{References}

[1] Baragiola R A., Planetary and Space Science, 51(14-15): 953-961(2003).

[2] Tachibana S, Kouchi A, Hama T, et al., Science advances, 3(9): eaao2538(2017).

[3] Symons M C R., Ultramicroscopy, 10(1-2): 97-103(1982).

[4] This work was performed at the Center for Nanoscale Materials, a U.S. Department of Energy Office of Science User Facility, and supported by the U.S. Department of Energy, Office of Science, under Contract No. DE-AC02-06CH11357. Yulin Lin is grateful for the Fundamental Research Funds for the Central Universities, China Scholarship Council (No201706270411). 